\title{
Tuberous sclerosis complex proteins control axon formation
}

\author{
Yong-Jin Choi, ${ }^{1}$ Alessia Di Nardo, ${ }^{1}$ Ioannis Kramvis, ${ }^{1}$ Lynsey Meikle, ${ }^{2}$ David J. Kwiatkowski, ${ }^{2}$ \\ Mustafa Sahin,, ${ }^{1,4}$ and $\mathrm{Xi} \mathrm{He}{ }^{1,3}$ \\ ${ }^{1}$ The F.M. Kirby Neurobiology Center, Department of Neurology, Children's Hospital Boston, Harvard Medical School, \\ Boston, Massachusetts 02115, USA; ${ }^{2}$ Division of Translational Medicine, Department of Medicine, Brigham and Women's \\ Hospital, Harvard Medical School, Boston, Massachusetts 02115, USA
}

\begin{abstract}
Axon formation is fundamental for brain development and function. TSC1 and TSC2 are two genes, mutations in which cause tuberous sclerosis complex (TSC), a disease characterized by tumor predisposition and neurological abnormalities including epilepsy, mental retardation, and autism. Here we show that Tsc1 and Tsc2 have critical functions in mammalian axon formation and growth. Overexpression of Tsc1/Tsc2 suppresses axon formation, whereas a lack of Tsc1 or Tsc2 function induces ectopic axons in vitro and in the mouse brain. Tsc2 is phosphorylated and inhibited in the axon but not dendrites. Inactivation of Tsc1/Tsc2 promotes axonal growth, at least in part, via up-regulation of neuronal polarity SAD kinase, which is also elevated in cortical tubers of a TSC patient. Our results reveal key roles of TSC1/TSC2 in neuronal polarity, suggest a common pathway regulating polarization/growth in neurons and cell size in other tissues, and have implications for the understanding of the pathogenesis of TSC and associated neurological disorders and for axonal regeneration.
\end{abstract}

[Keywords: Neuronal polarity; tuberous sclerosis complex, TSC; SAD kinase; autism]

Supplemental material is available at http://www.genesdev.org.

Received April 16, 2008; revised version accepted July 30, 2008.

Neuronal polarity, as defined by the elaboration of a single axon and multiple dendrites, is fundamental for brain development and function (Craig and Banker 1994; Arimura and Kaibuchi 2007). The axon and dendrites exhibit distinct cell biological compositions and functionality, and constitute the cellular basis for directional information flow within the nervous system /Craig and Banker 1994). Neuronal polarity has been studied using dissociated rat embryonic hippocampal neurons, which undergo characteristic growth stages in culture (Dotti et al. 1988; Craig and Banker 1994). These neurons initially form multiple lamellipodia after plating and generate minor neurites with similar lengths (stage 1-2); one of these neurites subsequently grows extensively to form an axon (stage 3), whereas the remaining neurites initiate subsequent growth and mature into dendrites (stage 4-5). This process appears to involve the initial axon fate determination coupled with polarized axonal growth and is reinforced by positive and negative feedback loops such that only a single axon is elaborated (Shi et al. 2003; Arimura and Kaibuchi 2007; Barnes et al. 2008). Many molecules have been identified to have a role in hippo-

Corresponding author.

${ }^{3}$ E-MAIL xi.he@childrens.harvard.edu; FAX (617) 730-1953.

${ }^{4}$ E-MAIL mustafa.sahin@childrens.harvard.edu; FAX (617) 730-1953.

Article is online at http://www.genesdev.org/cgi/doi/10.1101/gad.1685008. campal neuronal polarity in vitro (Shi et al. 2003; Menager et al. 2004; Nishimura et al. 2004; Schwamborn and Puschel 2004; Chen et al. 2006; Jacobson et al. 2006; Barnes et al. 2007). Of these, however, only LKB1 and SAD-A and SAD-B kinases have been shown to exert functions in neuronal polarity in vivo (Kishi et al. 2005; Barnes et al. 2007; Shelly et al. 2007).

Tuberous sclerosis complex (TSC) is an autosomal dominant hamartoma syndrome, which is characterized by dysplastic and disorganized overgrowth within many organs and is caused by loss-of-function mutations in TSC1 or TSC2 (Kwiatkowski and Manning 2005; Crino et al. 2006). TSC1 and TSC2 play central roles in restricting cell growth/size and therefore in tumor suppression (Tee et al. 2002; Inoki et al. 2003; Kwiatkowski and Manning 2005). TSC2 encodes a GTPase activating protein (GAP) for the Rheb GTPase, which is an activator for the mammalian TOR (target of rapamycin) kinase. mTOR in turn phosphorylates the ribosomal S6 kinase (S6K) and the translational regulator 4E-BP1, thereby stimulating the synthesis of selected proteins that promote cell growth (Wullschleger et al. 2006). The TSC1/TSC2 protein complex is obligatory for TSC2 GAP function, and together they inhibit mTOR signaling (Inoki et al. 2003; Stocker et al. 2003; Tee et al. 2003; Zhang et al. 2003). Growth factors stimulate cell growth/size through TSC2 phosphorylation and inactivation via the PI3 kinase 
Choi et al.

(PI3K) and the Akt kinase pathway (Inoki et al. 2002; Manning et al. 2002; Potter et al. 2002). In addition to hamartomas, most TSC patients exhibit neurological disorders including epilepsy, mental retardation, and autism, but the underlying pathological basis remains poorly defined. Here we show that TSC1 and TSC2 have critical negative roles in mammalian axon formation in vitro and in vivo.

\section{Results}

Tsc pathway components are expressed in neurons in a polarized manner

PI3K/Akt signaling in the axon may play a role in neuronal polarization in vitro (Shi et al. 2003; Jiang et al. 2005). Although Akt phosphorylates numerous targets, we considered the possibility that Akt may phosphorylate and inhibit TSC2 as observed in nonneuronal cells (Inoki et al. 2002; Manning et al. 2002; Potter et al. 2002). We first confirmed that in stage 3 hippocampal neurons in culture, the activated Akt (phospho-Akt, Ser473) was enriched in the axon as described (Fig. 1A,B; Shi et al.
2003). Although Tsc1 and Tsc 2 are localized in growth cones of cortical neurons in culture (Haddad et al. 2002), we found that, interestingly, the phosphorylated (at Thr1462) and thus inactivated Tsc2 was specifically localized along the axon (Fig. 1C,D), while total Tsc2 was evenly distributed throughout the neuron (Fig. 1E,F; Supplemental Fig. 1B). A PI3K inhibitor, LY294002, which blocks Akt activation and thus Tsc2 phosphorylation (Inoki et al. 2002), significantly reduced phosphoTsc2 staining (Supplemental Fig. 1A), implying that Tsc2 phosphorylation/inactivation in the axon is mediated by the PI3K/Akt pathway. Importantly, in mouse brain slices from embryonic day 19 (E19), the phospho-Tsc2 antibody exhibited indistinguishable staining from that of an axon-specific marker (SMI-312) in the axon-rich intermediate zone (Fig. 1I, arrows). We also found that phospho-S6K (Thr389), a downstream target of TSC/ mTOR signaling, was enriched in the axon but not in dendrites (Fig. 1G,H). Inhibition of mTOR via rapamycin markedly reduced the phospho-S6K staining in the axon (Supplemental Fig. 1C). These results suggest that inactivation of Tsc 2 and activation of the mTOR pathway occur specifically in the axon in polarized neurons.
Figure 1. Polarized activation/inactivation of Tsc pathway components in the axon versus dendrites. Axon-specific localization of active (phosphorylated) Akt, inactive (phosphorylated) Tsc2 and active (phosphorylated) S6K $(A, C, G)$ and the profiles of their fluorescence intensity $(B, D, H)$ in the axon and dendrites of stage 3 hippocampal neurons. Fixed neurons were costained with Taul (axon-specific marker) or Tuj1 (labeling both axon and dendrites). Each immunostaining was performed in at least four independent experiments, and the images shown are representative of more than 50 neurons examined. $(A, B)$ Phospho-Akt (S473) signal is enriched in the axon. $(C, D)$ Phospho-Tsc2 (T1462) signal is only detected in the axon (arrow), but not in dendrites (arrowheads). $(E, F)$ The level of Tsc2 is approximately the same in the axon and in dendrites. $(G, H)$ Phospho-S6K (T389) is enriched in the axon compared with the shorter dendrites. Bars, $10 \mu \mathrm{m}$. (I) PhosphoTsc2 staining is indistinguishable from axonal marker SMI-312 in the E19 cortical slice. Arrow indicates the main axon tracks positive for phosphoTsc2. Bar, $100 \mu \mathrm{m}$.
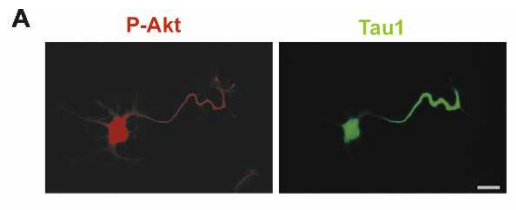

$$
\begin{aligned}
& \text { B } \\
& 30 \\
& 20 \\
& 100 \\
&
\end{aligned}
$$

C
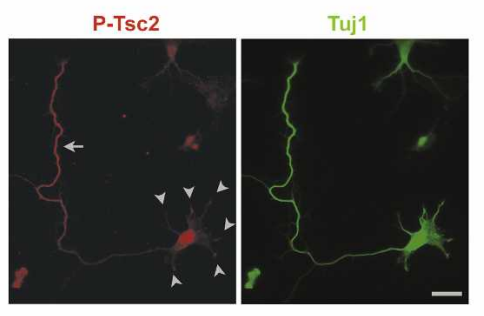

D
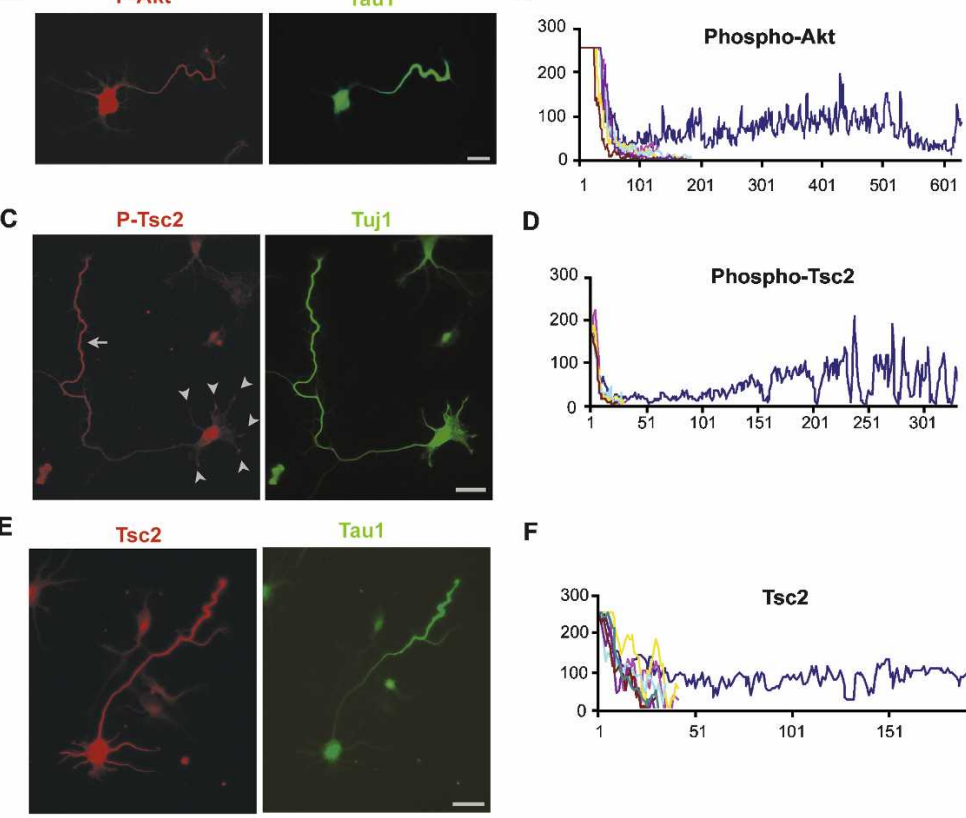

Tau1

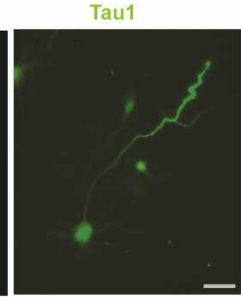

G P-S6K
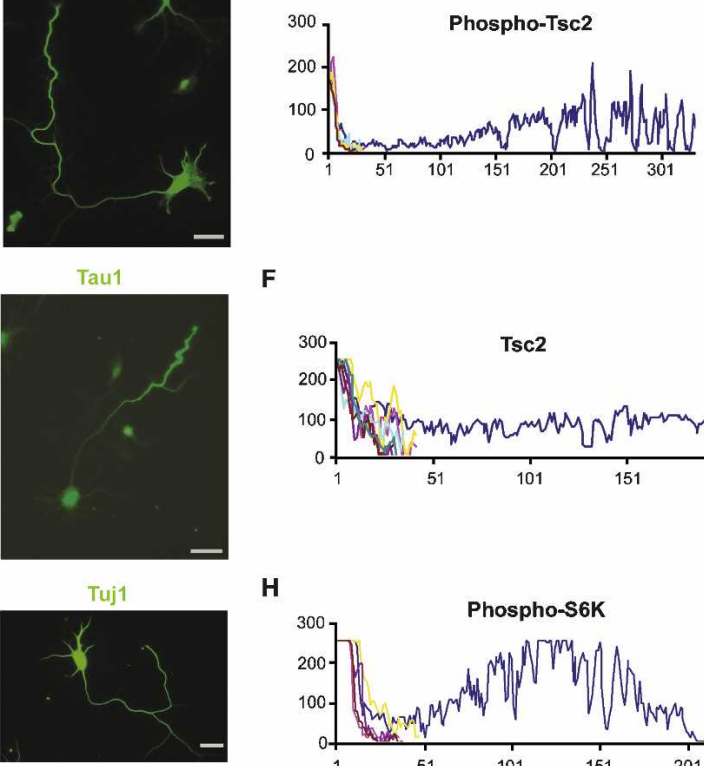

F

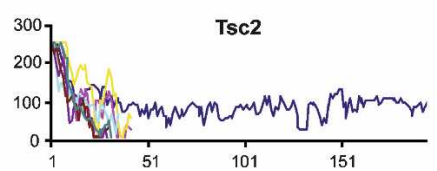

H

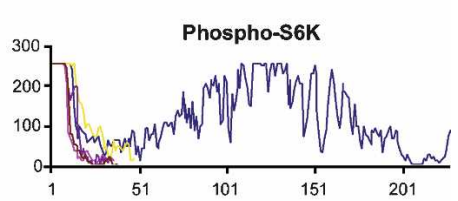

I P-Tsc2

SMI-312 Hoechst
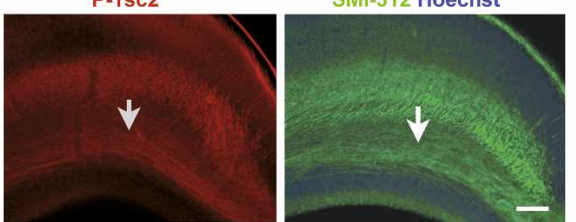

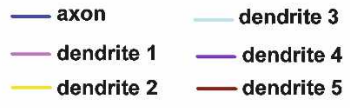


We further examined the localization of phospho-Tsc2 in earlier stages (stages 1-2) before and at the onset of neuronal polarization. At stage 1, phospho-Tsc2 was found to be concentrated in the cytoplasm in a punctuate manner and in lamellipodia (Supplemental Fig. 2). At stage 2, phospho-Tsc2 staining was prominent in the growth cone and the shaft of multiple neurites in the majority $(69.6 \%)$ of neurons, or, in contrast, was restricted to the growth cone and the shaft of a single neurite in the remaining $(30.4 \%)$ neurons (Supplemental Fig. 2 ). These two distinct patterns of phospho-Tsc2 at this early stage may reflect the polarization process by which a single neurite is selected to become the axon.

\section{The effect of Tsc overexpression and Tsc deficiency} on axon formation

We asked whether increasing Tsc1/Tsc2 expression would suppress axon specification and growth. Since Tsc1 and Tsc2 function as a heterodimeric complex, we coexpressed Tsc1 plus Tsc2 in hippocampal neurons. Tsc1/Tsc2 coexpression resulted in fewer neurons possessing an axon $159.2 \%$ of neurons, compared with $87.8 \%$ of neurons expressing EGFP) (Fig. 2A,B). Furthermore, $34.8 \%$ of neurons displayed no axons compared with $8.2 \%$ of neurons expressing EGFP (Fig. 2A,B). These results indicate that an increase in Tsc1/Tsc2 level inhibits axon growth.

We then examined the effect of loss of Tsc1/Tsc 2 on axon specification and growth in hippocampal neurons. We first studied the effect of Tsc2 knockdown using three different shRNA constructs that can each target both rat and mouse Tsc2 (Tsc2/sh1, Tsc2/sh2, and Tsc2/ sh3). These shRNAs significantly knocked down the expression of epitope-tagged and the endogenous Tsc2 in HEK293T cells and primary rat hippocampal neurons, respectively (Fig. 3A; Supplemental Fig. 3), and strikingly, resulted in an increase in neurons displaying multiple axons (from $9.6 \%$ to $43.6 \%$ ) that were positive for the axon-specific marker Taul (Fig. 3B).

We also generated $T s c 1^{-/-}$neurons via expressing Cre recombinase in cultured E17 hippocampal neurons isolated from Tsc1 conditional knockout mice (Tsc $1^{\text {flox } / \text { flox }}$ ) (Meikle et al. 2005, 2007). Cre effectively deleted the Tsc1 gene, as demonstrated by the reduced Tsc1 protein level (Fig. 3C). Tsc1 deletion also increased the number of neurons having multiple axons (from $4.3 \%$ to $45.8 \%$ ) (Fig. 3D). Neither the control EGFP (enhanced green fluorescence protein) expression in the Tsc1 flox/flox neurons (Fig. 3D) nor the Cre expression in the wildtype neurons (Supplemental Fig. 4) generated multiple axons. There was no statistically significant difference between $\mathrm{Tsc}^{-/-}$neurons and control neurons in terms of axonal and dendritic length (Supplemental Fig. 5). The indistinguishable effect of Tsc2 knockdown compared with Tsc1 deletion is consistent with Tsc1/Tsc2 functioning in a complex. These findings suggest that the Tsc1/Tsc2 protein complex restricts axon formation and growth.
A

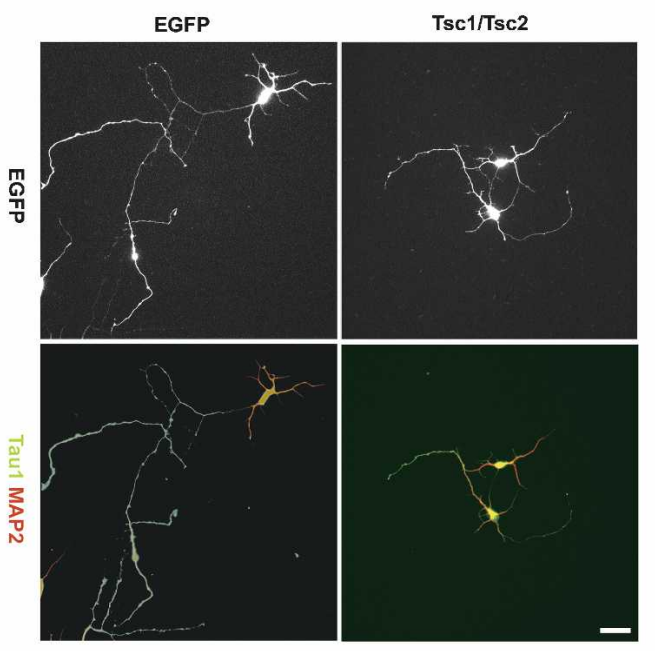

B

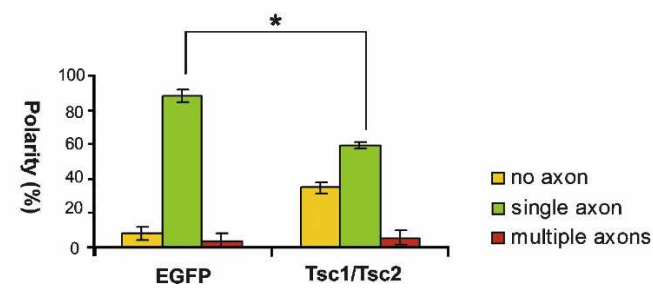

Figure 2. Tsc overexpression suppresses axon specification/ growth in culture. $(A, B)$ Suppression of axon formation by Tsc1 plus Tsc2 overexpression. (A) Representative hippocampal neurons transfected with EGFP alone or together with Tsc1/Tsc2. Neurons were fixed at 6DIV and stained with Taul (green, axonal marker) and MAP2 (red, dendritic marker). EGFP-expressing control cells show a single axon and multiple dendrites. Tsc1/Tsc2-expressing cells do not have a Tau1-positive process. $(B)$ Neuronal polarity phenotypes were categorized into three groups: no axon (yellow bar), single axon (green bar), and multiple axons (red bar). $\left({ }^{\star}\right) P<0.0005$ for single axon between the two groups by ANOVA. The data represent the mean \pm SD of 74-171 neurons per condition from three independent experiments. Bar, $20 \mu \mathrm{m}$.

\section{Tsc1/Tsc2 acts upstream of mTOR-mediated axonal growth regulatory pathway}

mTOR resides in two distinct multiprotein complexes called mTORC1 and mTORC2 (Sarbassov et al. 2005). mTORC1 is sensitive to rapamycin and controls cell growth through S6K and 4E-BP1, whereas mTORC2 is rapamycin-insensitive and regulates the actin cytoskeleton. We found that rapamycin prevented axonal growth but not dendritic growth in the wild-type hippocampal neurons (Fig. 4A). Furthermore, rapamycin treatment of Tsc2-deficient neurons not only decreased the number of neurons having multiple axons (from $54.7 \%$ to $13.5 \%$ ), but also increased the number of neurons without an axon (from $10.3 \%$ to $64.4 \%$ ) (Fig. 4A,B). Therefore, Tsc1/ Tsc2 controls axon formation via the mTORC1 pathway, consistent with phospho-S6K being enriched in the axon shaft (Fig. 1G,H). 
Choi et al.

Figure 3. TSC deficiency induces multiple axons in culture. $(A-D)$ Knockdown of Tsc2 by shRNA or knockout of Tsc1 induced multiple axons in vitro. $(A)$ Tsc2 protein level in E18 rat hippocampal neurons infected with a lentivirus expressing control shRNA or shRNA against Tsc2 (shTsc2). (B) E18 rat hippocampal neurons transfected with either EGFP alone or EGFP together with Tsc2 shRNA. Tsc2 knockdown induced multiple axons, all of which are positive for Taul. $\left(^{\star}\right) P<0.001$ for multiple axons between the two groups by ANOVA. (C) Tsc1 protein level in E17 Tsc1 flox/flox mouse hippocampal neurons infected with a control or Cre-expressing lentivirus. Neurons were lysed for Western blot analysis at 6DIV. (D) E17 mouse hippocampal neurons from Tsc1 flox/flox embryos transfected with EGFP alone or EGFP together with Cre. Arrowheads indicate axons positive for Taul. (*) $P<0.001$ for multiple axons between the two groups by ANOVA. The data represent the mean \pm SD of $135-185$ neurons per condition from three independent experiments. Bars, $20 \mu \mathrm{m}$.
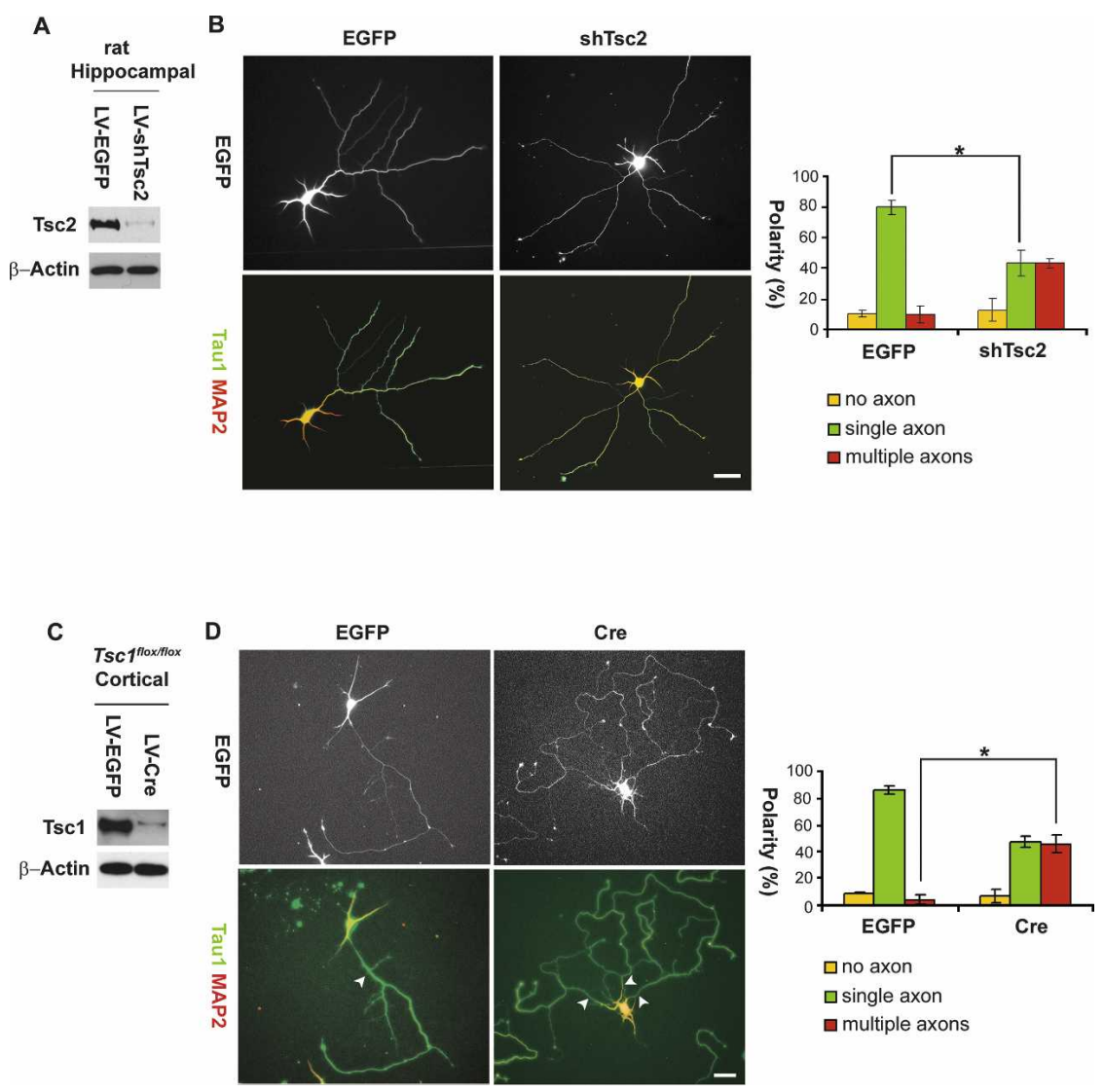

Tsc1 deficiency disrupts cortical neuronal morphology in vivo

We next asked whether a lack of Tsc function affects axon formation in vivo. We analyzed the brains of SynCre; Tsc1 $1^{\text {flox/flox }}$ mice (Meikle et al. 2007), in which Cre expression driven by the Synapsin-1 promoter results in deletion of the floxed Tsc1 alleles in post-mitotic neurons starting at E13 (Zhu et al. 2001). These Tsc1 mutant mice exhibit relatively normal cortical and hippocampal architecture, but develop neurological symptoms including seizures starting at postnatal day 5 (P5) and die between 3 and 5 wk after birth (Meikle et al. 2007). We analyzed $\mathrm{Tsc}^{-/-}$mutant cortices at P11 using two axonal markers (SMI-31 and SMI-312) (Xiao and Monteiro 1994; Shelly et al. 2007), together with a phospho-S6 antibody to mark cells exhibiting elevated mTOR signaling due to Tsc1 deletion (Fig. 5A). Both SMI-31 and SMI-312 staining was highly enriched in the axon-rich intermediate zone (Fig. 5A, arrowheads) in the control brain (Fig. 5A). Strikingly, however, Tsc1 $1^{-1-}$ cortices exhibited ectopic SMI-31- and SMI-312-positive axons throughout the cortex, including the upper cortical plate, which is normally rich in dendrites (Fig. 5A). These results suggest that a lack of Tscl function causes ectopic axon formation in the brain.

To further examine the role of $T s c 1 / 2$ proteins in neuronal morphogenesis at a single neuron resolution, we introduced either a control shRNA or a Tsc 2 shRNA together with EGFP into the cortical progenitors in E14E15 mouse embryos via electroporation as described (Barnes et al. 2007). Following electroporation, brain slices were prepared and incubated organotypically on tissue culture inserts for $3 \mathrm{~d}$ to allow enough time for the progenitors to differentiate and to migrate into the cortical plate. The neurons electroporated with the control shRNA showed typical morphology of migratory neurons, with $84 \%$ of the neurons exhibiting a thick apical leading process pointing toward pia and a slender trailing process (Fig. 5B,C; Noctor et al. 2004; Barnes et al. 2007). In contrast, $45 \%$ of the neurons electroporated with Tsc 2 shRNA displayed multiple processes oriented either laterally or basally (Fig. 5B,C), and exhibited retarded migration as reported in neurons with ectopic axon formation (Shelly et al. 2007). These data, together with the Tsc1 mutant phenotypes, suggest that Tsc/mTOR signaling is important for establishing neuronal polarity in vivo.

Tsc regulates the protein level of SAD kinases in neurons

In nonneuronal cells, the TSC-mTOR pathway controls cell size in part by selective translational regulation (Wullschleger et al. 2006). We did not observe general protein level changes between the wild-type and Tsc1/ 2-deficient hippocampal neurons (Fig. 6A; Supplemental 
A

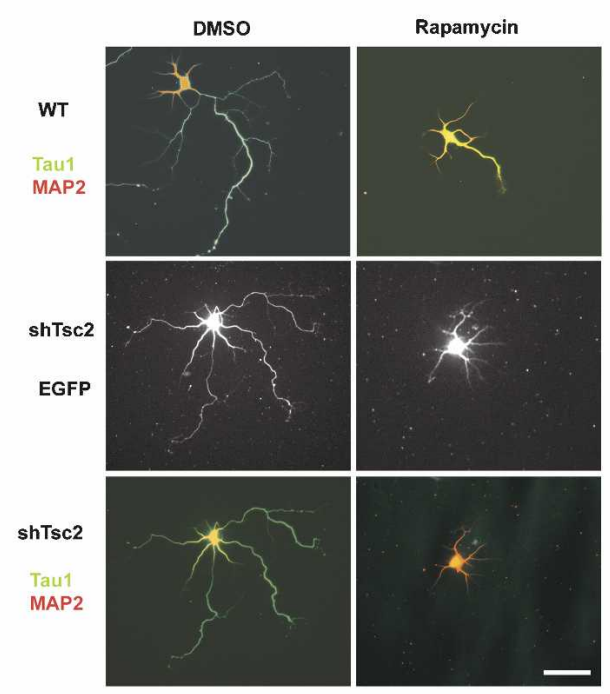

B

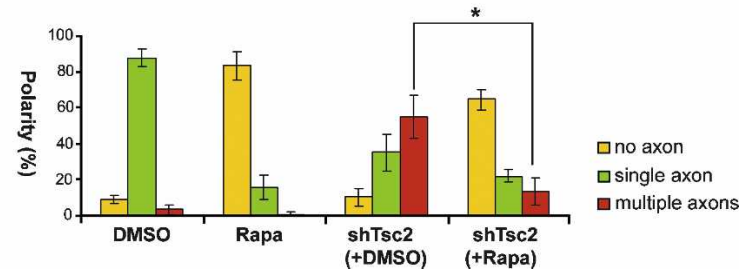

Figure 4. mTOR acts downstream from Tsc2 in neuronal polarity. (A) Rapamycin $(20 \mathrm{nM})$ inhibited axon formation/growth in both the wild-type (WT) and the Tsc2-knockdown rat hippocampal neurons. Bar, $20 \mu \mathrm{m}$. (B) Quantification of polarity defects. $\left(^{*}\right) P<0.01$ between multiple axons in shTsc2(+DMSO) and shTsc2(+Rapa) groups by ANOVA. The data represent the mean \pm SD of $127-180$ neurons per condition from three independent experiments.

Fig. 6). We searched for proteins that were regulated by Tsc1/2 during axon growth by focusing on molecules that have been implicated in neuronal polarity. Intriguingly, we found that Tsc2 knockdown specifically increased the protein level of the SAD kinase (Fig. 6A). SAD-A together with the related SAD-B have been shown to be critical for axon formation in the mouse brain (Kishi et al. 2005). The increase in SAD-A level by Tsc2 knockdown was dependent on mTORC1 activation and was prevented by rapamycin, which, of note, did not affect the basal SAD-A level (Fig. 6B). An elevated SAD-A protein level was also observed in $T s c 1^{-/-}$hippocampal and cortical neurons generated from Tsc1 flox/flox neurons expressing Cre (Fig. 6C). The increase in SAD-A protein amount in TSC1/2-deficient neurons was likely due to enhanced protein synthesis, because the SAD-A mRNA level was not altered upon Tsc2 knockdown (Supplemental Fig. 7A), and the SAD-A protein level was not affected when proteasome-dependent protein degradation was blocked in either the wild-type or the Tsc2deficient neurons (Supplemental Fig. 7B). We found that the SAD-B protein level was also elevated in $\mathrm{Tsc}^{-1-}$ cortical neurons (Supplemental Fig. 7C).
A vast majority of TSC patients exhibit neurological abnormalities (Crino et al. 2006) and harbor cortical tubers with characteristic "giant cells" that display high mTORC1 signaling and are readily identified by their elevated phospho-S6 levels (Baybis et al. 2004). We examined whether the SAD-A protein level is increased in cortical tubers resected from a TSC patient during epilepsy surgery. Phospho-S6-positive giant cells in the cortical tuber examined indeed displayed high SAD-A immunoreactivity, while the intensity of a control protein ( $\alpha$-tubulin) in those cells remained unchanged (Fig. 6E). These giant cells were also positive for neuronal marker MAP2 and NF (neurofilament), revealing their neuronal origin (Fig. 6E). We quantified sections double-stained with phospho-S6 and SAD-A and found that $60 \%$ of phospho-S6-positive cells had increased SAD-A protein. Taken together, the data produced in Tsc-deficient rat and mouse neurons as well as human cortical tubers demonstrate that loss of TSC function leads to increased SAD kinase protein expression.

It has been shown recently that LKB1 phosphorylates and activates SAD kinases, resulting in elevated Tau phosphorylation (at S262) (Barnes et al. 2007). We found that Tsc1-l- neurons exhibited increased levels of both phospho-SAD and phospho-Tau (S262) (Fig. 6D), suggesting that the elevated SAD protein level resulting from Tsc1 deficiency is accompanied by increased SAD kinase activity.

\section{$S A D$ kinases are required for Tsc regulation of axon formation}

We examined the role of SAD kinases in Tsc1/2-regulated axon growth. As SAD-A and SAD-B share significantly redundant roles (Kishi et al. 2005; Barnes et al. 2007), we used shRNAs that knocked down SAD-A and SAD-B, respectively, which were either expressed ectopically in HEK293T cells (Supplemental Fig. 8) or endogenously in hippocampal neurons (Supplemental Fig. 8). We found that knocking down SAD-A and SAD-B not only diminished axonal growth in wild-type neurons (Fig. 7A), but also inhibited the formation of multiple axons in Tsc2-deficient neurons (Fig. 7A). This inhibition was incomplete possibly due to residual SAD proteins or the existence of other potential targets regulated by Tsc1/Tsc2. These results, together with the finding of Tsc regulation of SAD-A and SAD-B synthesis, suggest that SAD kinases are important mediators of Tsc $1 / 2$ function in axon formation.

We also asked whether an increase in the SAD-A level could be sufficient for axon formation. SAD-A overexpression in hippocampal neurons resulted in two distinct phenotypes, as neurons with multiple axons (from $13.3 \%$ to $32.9 \%$ ) and those without an axon (from $5.5 \%$ to $24 \%$ ) were both increased in number (Fig. $7 \mathrm{~B}$ ). For neurons with multiple axons due to SAD-A overexpression, we did not observe significant changes in either axonal or dendritic length compared with control neurons expressing EGFP (Supplemental Fig. 9). We note that suppression of axon formation in some neurons by 
Choi et al.

Figure 5. Ectopic axon formation in SynCre; Tsc $1^{\text {flox/flox }}$ mouse cortex and defective neuronal polarization by Tsc 2 knockdown in organotypic brain slices. $(A)$ Control (left) and Tsc1 $^{-/-}$(right) cortical sections stained with phospho-S6 (green) and two axonal markers, SMI-31 or SMI-312 (red). Nuclei were stained by Hoechst (blue). In control mice (Syn-

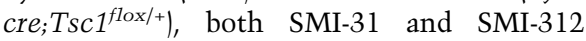
staining was concentrated in the axon-rich intermediate zone (IZ, arrowheads), but in Tsc1 ${ }^{-/}$mutant mice, SMI-31- and SMI-312positive processes were found throughout the cortex. Bars, $100 \mu \mathrm{m}$. (B) EGFP-positive mouse cortical neurons that express a control shRNA or Tsc2 shRNA and undergo radial migration in the intermediate zone. Computer-aided reconstruction/tracing of these neurons is shown below. E14-E15 mouse embryos were electroporated ex vivo with the shRNA constructs. Organotypic brain slices were cultured for $3 \mathrm{~d}$ in vitro. In contrast to the control neurons displaying a thick apical process pointing toward the pia, neurons electroporated with Tsc2 shRNA showed multiple processes oriented toward the lateral or basal direction. Bar, $50 \mu \mathrm{m}$. (C) Quantification of polarity defects in migrating cortical neurons in the intermediate zone. Phenotypes were categorized into three groups based on the orientation of the apical process: apical orientation within 15 degrees (green), lateral orientation between $15^{\circ}$ and $90^{\circ}$ (yellow), and basal orientation with $>90^{\circ}($ red). $\left.{ }^{\star}\right) P<0.0001$ by $\chi^{2}$ test. The data represent the mean of $79-83$ neurons per condition from three separate experiments.

SAD-A overexpression is not entirely surprising and is reminiscent of similar observations previously made by overexpression of other polarity proteins such as Par6 (Shi et al. 2003; Nishimura et al. 2005), and may imply that SAD kinases function in concert with other molecules to promote axonal polarity.

\section{Discussion}

In this study, we provide evidence that the Tsc $1 / 2$ protein complex is required for neuronal polarity/axon formation. We find that cortical and hippocampal neurons deficient for Tsc1/2 function form ectopic axons both in vitro and in vivo. We further identify neuronal polarity SAD kinases, particularly SAD-A, as target proteins, the synthesis of which is regulated by $T s c 1 / 2$ in neurons. Thus loss of Tsc function is associated with an increase in SAD kinase protein levels in rodent neuronal cultures and in cortical tubers from a TSC patient. In addition, we find that suppressing SAD expression partially rescues the multipolar neuronal phenotype in Tsc1/2-deficient neurons. Thus, the Tsc/mTOR pathway appears to limit multiple axon formation and acts to confine polarized growth within a single axon in the mammalian brain, and its deregulation likely contributes to the neurological symptoms commonly observed in TSC patients.

Axon formation in the brain is governed by extracellular signals and the intrinsic polarization apparatus (Arimura and Kaibuchi 2007; Barnes et al. 2008). Tsc1/ Tsc2 is ideally suited to couple extracellular signals to the intracellular growth machinery (Wullschleger et al. 2006). We observed that Tsc 2 is phosphorylated, and thus inhibited, and the mTORC1 pathway is activated in the axon. Inactivation of Tsc1/Tsc2 allows the prospective axon to initiate and maintain its growth through mTORC1, while Tsc1/Tsc2 keeps the growth of the remaining neurites/dendrites on hold. Growth factors such as IGF-1 (insulin-like growth factor-1) and BDNF (brain derived growth factor) (Yoshimura et al. 2005; Sosa et al. 2006; Shelly et al. 2007) activate PI3K and Akt, which phosphorylate and inactivate Tsc2 (Inoki et al. 2002; Manning et al. 2002; Potter et al. 2002). Furthermore, Wnt inhibition of GSK3, which phosphorylates and activates Tsc2, also leads to Tsc2 inactivation (Inoki et al. 2006). Thus Tsc 2 regulation may mediate the capability of IGF, BDNF, and Wnt in specifying neuronal polarity and axonal morphogenesis (Hilliard and Bargmann 2006; Sosa et al. 2006; Shelly et al. 2007). We note that it has been suggested that Akt promotes axon formation by phosphorylation and inhibition of GSK3 (Jiang et al. 2005; Yoshimura et al. 2005). However, mice harboring GSK3 knockin alleles that abolish the Akt phosphorylation site are viable and fertile, and cortical and hippocampal neurons of these mice are polarized normally in vitro and in vivo (McManus et al. 2005; Gartner et al. 2006). These and our results together suggest that Akt phosphorylation of Tsc2, but not GSK3, likely plays a critical role in neuronal polarity/axonal growth.

While phosphorylation and inactivation of Tsc2 is observed in the axon in polarized neurons, at early stages (or the onset) of neuronal polarization, phosphorylated Tsc2 (Thr1462) is observed in the growth cone and shaft of multiple neurites in a majority $(70 \%)$ of neurons, and interestingly, also in the growth cone and shaft of only a single neurite in the remaining $(30 \%)$ neurons. We interpret these two distinct patterns of phosphorylated Tsc2 


\section{A}
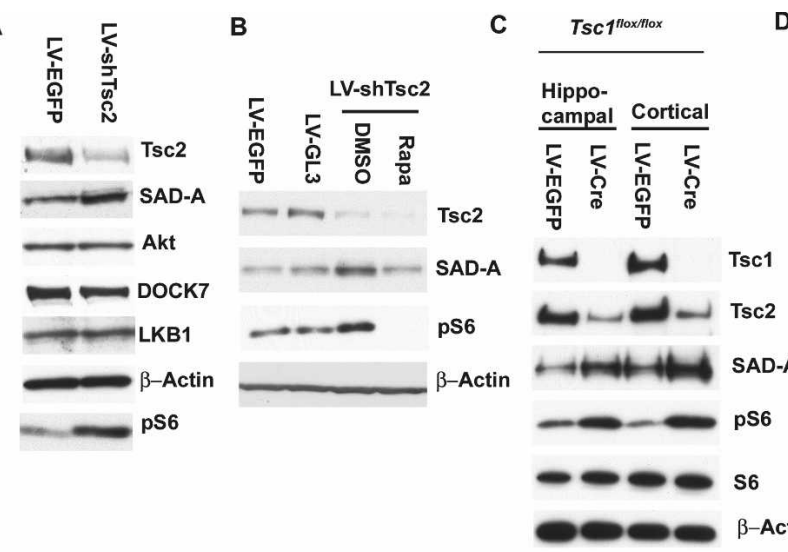

E
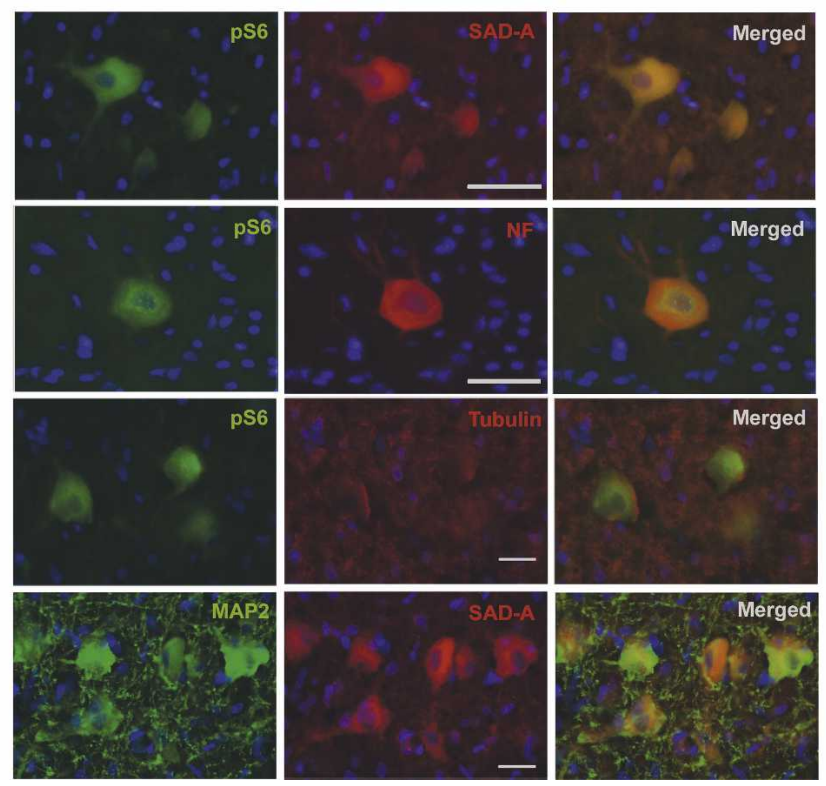

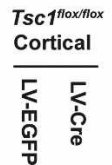

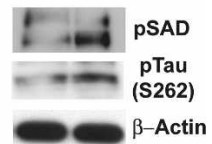

\section{-A} $\beta$-Actin

(1)

Figure 6. The SAD-A kinase protein level is regulated by $\mathrm{Tsc} 1 / \mathrm{Tsc} 2$ in hippocampal and cortical neurons and in cortical tubers of a TSC patient. (A) Rat hippocampal neurons infected with a lentivirus expressing EGFP (LV-EGFP) or an shRNA against Tsc2 (LV-shTsc2). Neurons were lysed at 6DIV for Western blot analysis. Tsc2 knockdown increased the levels of phospho-S6 and the SAD-A protein level. $(B)$ Increase in SAD-A protein level upon Tsc2-knockdown was blocked by rapamycin treatment. Neurons were infected with a control lentivirus (LV-EGFP and LV-GL3, shRNA against luciferase) or LVshTsc2. Cells were treated with either DMSO or rapamycin $(20 \mathrm{nM})$, and lysates were made at 6DIV for Western blot analysis. Note that the basal level of SAD-A protein was not affected by rapamycin. $(C)$ The SAD-A protein level was increased upon deletion of the conditional Tsc1 $1^{\text {flox/flox }}$ alleles in vitro. E17 Tsc1 flox/flox hippocampal and cortical neurons were transduced with a lentivirus expressing EGFP or Cre. Note that Tsc1 knockout also markedly reduced the level of Tsc2 protein as reported and increased phospho-S6 (pS6). (D) Deletion of Tsc1 $1^{\text {flox/flox }}$ alleles in cultured neurons resulted in increased levels of phospho-SAD and phospho-Tau (S262). Phospho-SAD was detected using the pSAD(T-al) antibody, which recognizes both phosphoSAD-A and -B. (E) Immunohistochemical analysis of SAD-A expression in human tubers from a TSC patient. Representative images of resected brain sections show colocalization of (green) phospho-S6 (pS6) and (red) SAD-A in the giant cells of the tuber lesion. Positive SAD-A staining was identified in $60 \%$ of the pS6-positive giant cells (counted 79/131). The giant cells expressing a high level of SAD-A protein were also positive for neuronal marker MAP2. (Red) Neurofilament staining (NF) confirms increased mTOR activity in the enlarged pS6-positive dysmorphic cells as described (Ozcan et al. 2008). The staining of control protein $\alpha$-tubulin showed no changes in immunoreactivity in pS6-positive cells. Hoechst dye staining for DNA is shown in blue. Bars, $50 \mu \mathrm{m}$.

as a reflection of neurons in the polarization process through which a single neurite is selected to become the axon. While deletion or knockdown of Tscl/2 is sufficient to initiate and maintain multiple axon formation, transient phosphorylation/inhibition of Tsc2, as observed in multiple neurites in stage 2 neurons, does not lead to supernumeric axons. It seems likely that persistent and prolonged phosphorylation/inhibition of Tsc2 in a single neurite, as a result of positive and negative feedback mechanisms (Shi et al. 2003; Arimura and Kaibuchi 2007; Barnes et al. 2008), leads to axon specification and growth. Given the critical role of Tsc/mTOR signaling in regulation of protein synthesis, persistent phosphorylation/inhibition of Tsc2 and activation of mTOR may result in accumulation of key proteins such as SAD kinases (see below) for axonogenesis. The mecha- nisms by which phosphorylated/inactivated Tsc2 is restricted from multiple neurites to a single neurite (the future axon) and is maintained in the axon deserve further investigation.

What downstream effectors are regulated by Tsc/ mTOR signaling during neuronal polarization? In our study, we found that the synthesis of SAD proteins is specifically up-regulated by the inactivation of Tsc and the activation of mTOR signaling. SAD kinases have been shown as important regulators of neuronal polarity both in vitro and in vivo (Kishi et al. 2005). Recently, LKB1 was identified as a critical upstream kinase and activator for SAD kinases, suggesting a kinase cascade for axon formation (Barnes et al. 2007). We found that in $T s c 1^{-/-}$neurons, the elevated SAD protein amount is accompanied by elevated levels of phosphorylated SAD 
Choi et al.

Figure 7. Effects of SAD-A/B knockdown on axon specification/growth in vitro. (A) Rat hippocampal neurons were transfected with control shRNA, SAD-A and SAD-B shRNAs (shSAD-A/B), Tsc2 shRNA (shTsc2), and shTsc2 together with shSAD-A/B, and stained with MAP2 (dendritic marker) and SMI-312 (axonal marker). Knockdown of SAD-A/B blocked the multiple-axon phenotype induced by the Tsc2 shRNA. Quantification is shown on the right. $\left(^{\star}\right) P=0.001$ for multiple axons between the shTsc2 and shTsc2 + shSAD-A/B groups by ANOVA. $(B)$ Effects of SAD-A overexpression on neuronal polarity. Rat hippocampal neurons expressing EGFP alone or expressing EGFP together with SAD-A were fixed at 6DIV. SAD-A overexpression induced multiple axons in some neurons and inhibited axon formation in others. Quantification is shown on the right. $\left(^{*}\right)$ $P<0.05$ for multiple axons between the two groups by ANOVA. Bars, $20 \mu \mathrm{m}$. The data represent the mean \pm SD of 123-142 neurons per condition from three independent experiments.
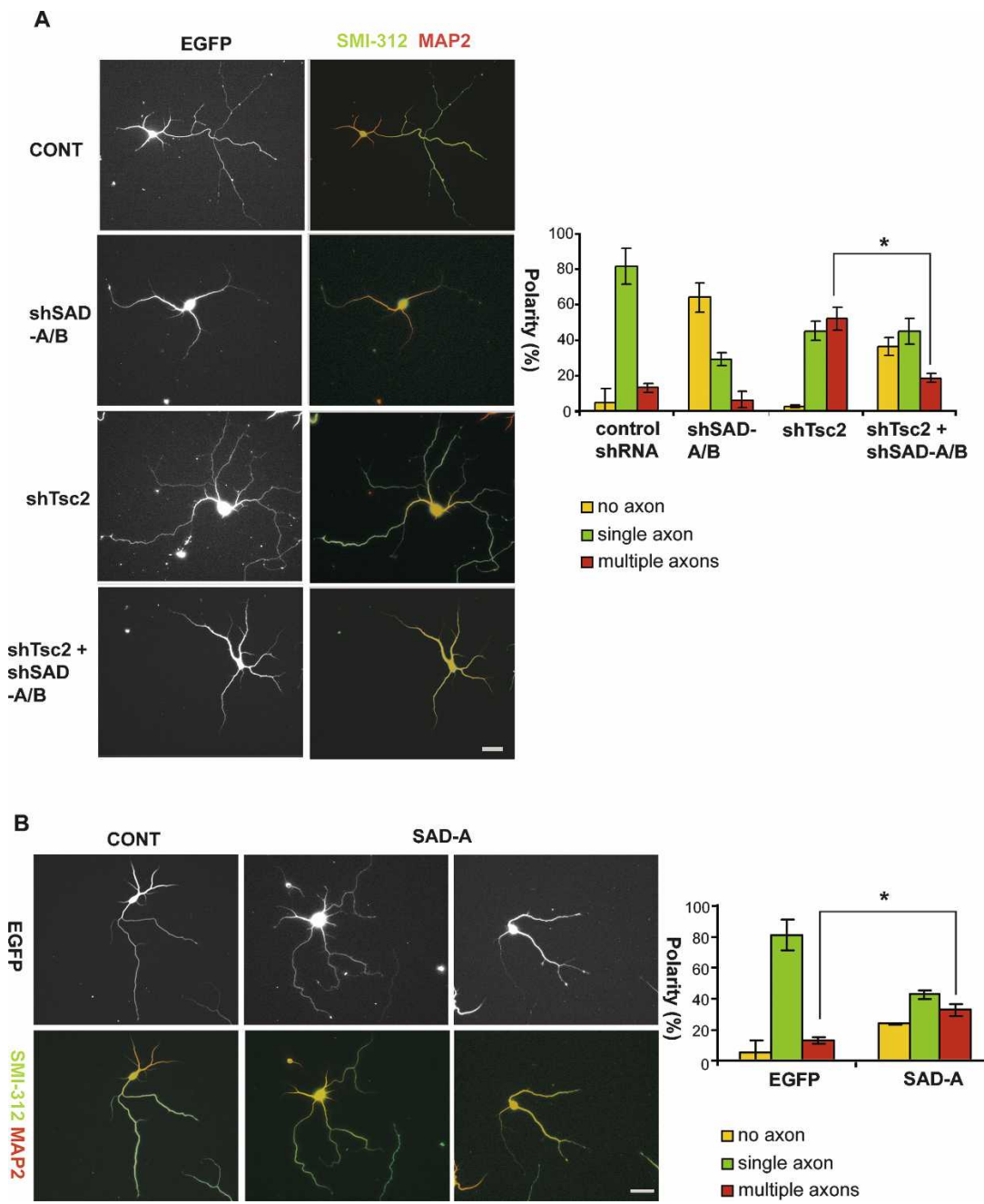

and phosphorylated Tau, which is a SAD substrate (Kishi et al. 2005). These results demonstrate increased SAD activity and imply that LKB1 and/or other kinases that phosphorylate SAD proteins are active in these neurons. Concerted control of the SAD protein amount and activity by Tsc1/Tsc2 and LKB1 (and/or other kinases), respectively, may be pivotal for the integration of axon specification and growth. We note that in cancer cells, LKB1 can activate Tsc1/Tsc2 via the AMPK kinase under energy stress conditions (Shaw et al. 2004a,b). However, in axonogenesis, Tsc1/2 and LKB1 deficiencies have opposite effects. Loss of Tsc1/2 results in neurons with multiple axons, while loss of LKB1 results in neurons with no axons. Therefore, LKB1 activation of $\mathrm{Tsc} 1 / 2$ is unlikely to be involved in regulating axonal growth. Although we identified SAD proteins as targets of Tsc/mTOR signaling, the fact that SAD-A/B knockdown only partially rescues the multiaxon phenotype implies that there are likely other downstream targets yet to be identified. Future studies will shed light on novel mechanisms regulated by the Tsc/mTOR pathway during axon formation and expand our understanding of the various causes of clinical manifestations of TSC in the brain and other organs. In addition, our study suggests that manipulating the TSC pathway may have therapeutic applications to axonal repair/regeneration after neuronal injuries.

\section{Materials and methods}

\section{Primary neuronal culture and transfection}

Hippocampal cells were prepared according to published protocols (Sahin et al. 2005). Briefly, hippocampi isolated from either rat (E18) or mouse (E17) were digested with papain for $10 \mathrm{~min}$ at $37^{\circ} \mathrm{C}$ in Hank's balanced salt solution buffered with HEPES. Cells were dissociated in plating medium (Neurobasal medium supplemented with B27) by trituration with a pipette. Neurons were transfected immediately after dissociation using Amaxa Nucleofactor (Amaxa Biosystems) and plated on the chamber slides. Transfected neurons were plated on the Lab-Tek II chamber slides (Nunc) coated with poly-D-Lysine ( $1 \mathrm{mg} / \mathrm{mL}$; Sigma) at a density of 1000 cells per well $\left(1.7 \mathrm{~cm}^{2}\right)$. After cells attach to the slides, medium was replaced with fresh plating medium to remove unattached cells. 


\section{Ex vivo electroporation and organotypic slice culture}

Two to three microliters of DNA constructs $(2 \mu \mathrm{g} / \mu \mathrm{L})$ together with EGFP plasmid (2:1 ratio) was injected into the lateral ventricle of each harvested mouse embryo (E14-E15) using a microinjector (PLI-100; Medical Systems Corp). Fastgreen (0.3 mg/ $\mathrm{mL}$ ) was added to the DNA solution to monitor the location and the amount of injection. The head of each injected embryo was electroporated (four 100-msec pulses, $40 \mathrm{~V}, 100$-msec interval) using Tweezertrode (Harvard Apparatus) coupled with an electroporator (ECM 830, BTX). Electroporated brains were dissected out and embedded in the $3 \%$ low-melting agarose at $\sim 38^{\circ} \mathrm{C}$. Upon solidification on ice, $250-\mu \mathrm{m}$ coronal brain sections were made by vibratome (Leica VT100S), and the slices were transferred onto the tissue culture inserts (Millicell; Millipore) bathing on culture medium (Neurobasal medium with B27, N2, L-glutamine, and penicillin and streptomycin) in sixwell plates. After washing with culture medium, slices were incubated organotypically on the inserts for $3 \mathrm{~d}$. All procedures were carried out in accordance with the Guide for the Humane Use and Care of Laboratory Animals, and the study was approved by the Animal Care and Use Committee of Children's Hospital Boston.

\section{Quantification of morphology defects in migrating cortical neurons}

The morphology of individual migrating cortical neurons in the intermediate zone was categorized into three groups based on the orientation of their processes. An apical process oriented within $15^{\circ}$ from a line orthogonal to the pial surface was considered normal. A process oriented more than $15^{\circ}$ was grouped into either lateral or basal. A $\chi^{2}$ test was used for the statistical analysis.

\section{DNA constructs}

pcDNA3-Tsc1 and Tsc2 constructs were generously provided by K.-L. Guan (Inoki et al. 2002). SAD-A and SAD-B constructs were generously provided by J. Sanes (Kishi et al. 2005). Lentiviral vectors were gifts from R. Mulligan (Mostoslavsky et al. 2005). To generate lentiviral Cre constructs, the Cre cDNA was amplified with a nuclear localization signal using PCR, and then cloned into lentiviral vectors. The lentiviral Tsc2 shRNA construct was custom made by Cellogenetics. The target sequences are as follows: Tsc2/sh1 (5'-GGTGAAGAGAGCCG TATCACA-3 $\left.{ }^{\prime}\right), \quad$ Tsc2/sh2 (5'-GGTGTCATATGAGATTGT TCT- $3^{\prime}$ ), and Tsc2/sh3 (5'-GCACGGAGCACCAGTCTTAAT$3^{\prime}$ ). Tsc2/sh1 was adapted from a published sequence (Tavazoie et al. 2005). Lentiviral shRNA constructs against SAD-A and SAD-B were purchased from Sigma.

\section{Antibodies and inhibitors}

The following antibodies and inhibitors were used: anti-Akt, anti-phospho-Akt (S473), anti-phospho-Tsc2 (T1462), anti-Tsc1, anti-phospho-S6K (T389), anti-phospho-S6 (S235/236; Cell Signaling); anti-Taul, anti-MAP2 (Chemicon); anti- $\beta$-Actin (Sigma); anti-Tsc2 (Upstate); anti- $\alpha$-Tubulin (Santa Cruz); antiTuj1, SMI-31, SMI-312 (Covance); anti-phospho-Tau (S262; Invitrogen); anti-MAP-2 (Santa Cruz); Alexa 350-conjugated goat anti-mouse/rabbit, Alexa 568-conjugated goat anti-mouse/rabbit, Alexa 488-conjugated goat anti-mouse/rabbit (Invitrogen); LY294002 (PI-3 kinase inhibitor), Rapamycin (mTOR inhibitor), MG-132 (proteasome inhibitor) (Calbiochem). Anti-SAD-A,
anti-SAD-B, and anti-Phospho-SAD(T-al) antibodies were kindly provided by J. Sanes (Kishi et al. 2005; Barnes et al. 2007).

\section{Immunocytochemistry and immunohistochemistry}

Neurons were fixed with $4 \%$ paraformaldehyde for $10 \mathrm{~min}$ and washed with PBS. Fixed neurons were permeablized and blocked with a solution containing $3 \%$ goat serum, $3 \%$ BSA, and $0.1 \%$ Triton X-100 in PBS. Neurons were incubated with primary antibody overnight at $4^{\circ} \mathrm{C}$. After washing with PBS, cells were stained with secondary antibodies at room temperature for $1 \mathrm{~h}$ and mounted with anti-fading agent (Aqua-poly mount; Polysciences). Mice were anesthetized and perfused with PBS, followed by $4 \%$ paraformaldehyde in PBS. Fixed brains were then cryoprotected in $30 \%$ sucrose in PBS. Coronal sections $(50 \mu \mathrm{m})$ were cut using a Microm K400 Fast Freezing Unit and HM450 Sliding Electronic Microtome and stored in PBS at $4{ }^{\circ} \mathrm{C}$ until use. Cortical sections were blocked in PBS containing $4 \%$ goat serum and $0.1 \%$ Triton X-100 for $2 \mathrm{~h}$ and were incubated with primary antibodies in blocking solution overnight at $4^{\circ} \mathrm{C}$. After wash, floating sections were incubated with secondary antibodies and mounted with Aqua-poly mount (Polysciences). Surgical resection specimens were fixed in 4\% paraformaldehyde, and $16-\mu \mathrm{m}$ serial sections were prepared using a cryostat. Sections were incubated overnight at $4^{\circ} \mathrm{C}$ with antibodies. Monoclonal antibody against SAD-A (1:100), polyclonal antibody against phospho-S6 (1:300), monoclonal antibody against SMI-311 (1:100), monoclonal antibody against $\alpha$ tubulin (1:500), and polyclonal antibody against MAP-2 (1:800) were used. Subsequently, sections were stained with fluorescent-conjugated secondary antibodies. Negative controls were taken at the same exposure. Work on human tissues was approved by the Committee on Clinical Investigation at Children's Hospital Boston.

\section{Image analysis}

Images were obtained with Hamamatsu ORCA-ER camera using IPLab software. Fluorescence intensities were measured using Metamorph software. Neuronal polarity phenotypes were categorized into three groups (no axon, single axon, and multiple axons). An axon was defined as a neurite whose length is three times longer than other neurites and is also immunoreactive for axonal markers (Taul or SMI-312). Cells were then scored in a blinded manner. Statistical analysis of neuronal polarity defects in neurons was carried out by ANOVA. To examine neuronal polarity of Syn-Cre; Tsc1 $1^{\text {flox/flox }}$ mice, a Z series of cortical sections stained with SMI-31/phospho-S6 or SMI-312/ phospho-S6 was collected using a Zeiss LSM510 Confocal Scanning Laser microscope, and a projection was generated using LSM software.

\section{Lentivirus generation and infection}

Lentiviral supernatants were prepared by cotransfecting 293T cells with each lentiviral vector and four packaging vectors (rev, tat, gag/pol, vsv-g) using Trans-IT-293T transfection reagent (Mirus Bio). After overnight incubation, medium was replaced and the supernatants were collected $48-60 \mathrm{~h}$ after transfection. Dissociated hippocampal neurons were plated on six-well plates coated with poly-D-Lysine at a density of $1 \times 10^{6}$ cells/well of a six-well plate. After $6 \mathrm{~h}$, neurons were infected with lentiviral supernatants overnight. Neurons were washed three times with plating medium (Neurobasal medium supplemented with B27) and incubated for another $5 \mathrm{~d}$. 
Choi et al.

\section{$R T-P C R$}

To prepare RNA, rat hippocampal neurons were lysed at 6DIV, and RNAs were prepared using an RNeasy minikit (Qiagen) according to the manufacturer's protocol. First-strand synthesis was carried out using M-MLV reverse transcriptase (Invitrogen), and $1.5 \mu \mathrm{L}$ from each reaction was used as a template for each PCR reaction. The following primers were used for PCR: SAD-A (5'-TTGAAGCCAGAGAACCTGCT-3', 5' -GCTTGACCTTCT CCAGCAAC- $\left.3^{\prime}\right)$, Tsc2 (5'-CTGGATGCTGTGGTCTGCTA$3^{\prime}, 5^{\prime}$-CACAGATGTGGGGGAGTTCT-3'), and b-Actin $\left(5^{\prime}\right.$ AAGACCTCTATGCCAACACAG-3', 5'-CTGCTTGCTGAT CCACATCTG-3').

\section{Acknowledgments}

We thank Joshua Sanes and Linda Van Aelst for SAD-A/SADB/phospho-SAD(T-al) and DOCK7 antibodies, respectively; Kun-Liang Guan and J. Sanes for expression constructs; Li-Hui Tsai for protocols for cortical slices; June Goto, Namjik Cho, and Abbey Sadowski for technical help; and Larry Benowitz, Mike Greenberg, and Zhigang He for comments. Y-J.C. and A.D.N are in part supported by grants from the Hearst Fund. M.S. acknowledges support from the Tuberous Sclerosis Alliance, Manton Foundation, and a grant from Children's Hospital Boston Mental Retardation and Developmental Disability Research Center (P01HD18655). This work is in part supported by NIH grant NS031535 to D.J.K, and NS058956 to M.S., who is an American Academy of Neurology/SMA Young Investigator; and to X.H., who is a Leukemia and Lymphoma Society Scholar.

\section{References}

Arimura, N. and Kaibuchi, K. 2007. Neuronal polarity: From extracellular signals to intracellular mechanisms. Nat. Rev. Neurosci. 8: 194-205.

Barnes, A.P., Lilley, B.N., Pan, Y.A., Plummer, L.J., Powell, A.W., Raines, A.N., Sanes, J.R., and Polleux, F. 2007. LKB1 and SAD kinases define a pathway required for the polarization of cortical neurons. Cell 129: 549-563.

Barnes, A.P., Solecki, D., and Polleux, F. 2008. New insights into the molecular mechanisms specifying neuronal polarity in vivo. Curr. Opin. Neurobiol. 18: 44-52.

Baybis, M., Yu, J., Lee, A., Golden, J.A., Weiner, H., McKhann II, G., Aronica, E., and Crino, P.B. 2004. mTOR cascade activation distinguishes tubers from focal cortical dysplasia. Ann. Neurol. 56: 478-487.

Chen, Y.M., Wang, Q.J., Hu, H.S., Yu, P.C., Zhu, J., Drewes, G., Piwnica-Worms, H., and Luo, Z.G. 2006. Microtubule affinity-regulating kinase 2 functions downstream of the PAR-3/ PAR-6/atypical PKC complex in regulating hippocampal neuronal polarity. Proc. Natl. Acad. Sci. 103: 8534-8539.

Craig, A.M. and Banker, G. 1994. Neuronal polarity. Annu. Rev. Neurosci. 17: 267-310.

Crino, P.B., Nathanson, K.L., and Henske, E.P. 2006. The tuberous sclerosis complex. N. Engl. J. Med. 355: 1345-1356.

Dotti, C.G., Sullivan, C.A., and Banker, G.A. 1988. The establishment of polarity by hippocampal neurons in culture. $J$. Neurosci. 8: 1454-1468.

Gartner, A., Huang, X., and Hall, A. 2006. Neuronal polarity is regulated by glycogen synthase kinase-3 (GSK-3 $\beta$ ) independently of Akt/PKB serine phosphorylation. J. Cell Sci. 119: 3927-3934.

Haddad, L.A., Smith, N., Bowser, M., Niida, Y., Murthy, V., Gonzalez-Agosti, C., and Ramesh, V. 2002. The TSC1 tumor suppressor hamartin interacts with neurofilament-L and possibly functions as a novel integrator of the neuronal cytoskeleton. J. Biol. Chem. 277: 44180-44186.

Hilliard, M.A. and Bargmann, C.I. 2006. Wnt signals and frizzled activity orient anterior-posterior axon outgrowth in C. elegans. Dev. Cell 10: 379-390.

Inoki, K., Li, Y., Zhu, T., Wu, J., and Guan, K.L. 2002. TSC2 is phosphorylated and inhibited by Akt and suppresses mTOR signalling. Nat. Cell Biol. 4: 648-657.

Inoki, K., Li, Y., Xu, T., and Guan, K.L. 2003. Rheb GTPase is a direct target of TSC2 GAP activity and regulates mTOR signaling. Genes \& Dev. 17: 1829-1834.

Inoki, K., Ouyang, H., Zhu, T., Lindvall, C., Wang, Y., Zhang, X., Yang, Q., Bennett, C., Harada, Y., Stankunas, K., et al. 2006. TSC2 integrates Wnt and energy signals via a coordinated phosphorylation by AMPK and GSK3 to regulate cell growth. Cell 126: 955-968.

Jacobson, C., Schnapp, B., and Banker, G.A. 2006. A change in the selective translocation of the Kinesin-1 motor domain marks the initial specification of the axon. Neuron 49: 797804.

Jiang, H., Guo, W., Liang, X., and Rao, Y. 2005. Both the establishment and the maintenance of neuronal polarity require active mechanisms: Critical roles of GSK-3 $\beta$ and its upstream regulators. Cell 120: 123-135.

Kishi, M., Pan, Y.A., Crump, J.G., and Sanes, J.R. 2005. Mammalian SAD kinases are required for neuronal polarization. Science 307: 929-932.

Kwiatkowski, D.J. and Manning, B.D. 2005. Tuberous sclerosis: A GAP at the crossroads of multiple signaling pathways. Hum. Mol. Genet. 14: R251-R258. doi: 10.1093/hmg/ ddi260.

Manning, B.D., Tee, A.R., Logsdon, M.N., Blenis, J., and Cantley, L.C. 2002. Identification of the tuberous sclerosis complex-2 tumor suppressor gene product tuberin as a target of the phosphoinositide 3-kinase/akt pathway. Mol. Cell 10: 151-162.

McManus, E.J., Sakamoto, K., Armit, L.J., Ronaldson, L., Shpiro, N., Marquez, R., and Alessi, D.R. 2005. Role that phosphorylation of GSK3 plays in insulin and Wnt signalling defined by knockin analysis. EMBO J. 24: 1571-1583.

Meikle, L., McMullen, J.R., Sherwood, M.C., Lader, A.S., Walker, V., Chan, J.A., and Kwiatkowski, D.J. 2005. A mouse model of cardiac rhabdomyoma generated by loss of Tsc1 in ventricular myocytes. Hum. Mol. Genet. 14: 429-435.

Meikle, L., Talos, D.M., Onda, H., Pollizzi, K., Rotenberg, A., Sahin, M., Jensen, F.E., and Kwiatkowski, D.J. 2007. A mouse model of tuberous sclerosis: Neuronal loss of Tsc1 causes dysplastic and ectopic neurons, reduced myelination, seizure activity, and limited survival. J. Neurosci. 27: 55465558.

Menager, C., Arimura, N., Fukata, Y., and Kaibuchi, K. 2004. PIP3 is involved in neuronal polarization and axon formation. J. Neurochem. 89: 109-118.

Mostoslavsky, G., Kotton, D.N., Fabian, A.J., Gray, J.T., Lee, J.S., and Mulligan, R.C. 2005. Efficiency of transduction of highly purified murine hematopoietic stem cells by lentiviral and oncoretroviral vectors under conditions of minimal in vitro manipulation. Mol. Ther. 11: 932-940.

Nishimura, T., Kato, K., Yamaguchi, T., Fukata, Y., Ohno, S., and Kaibuchi, K. 2004. Role of the PAR-3-KIF3 complex in the establishment of neuronal polarity. Nat. Cell Biol. 6: 328-334.

Nishimura, T., Yamaguchi, T., Kato, K., Yoshizawa, M., Nabeshima, Y., Ohno, S., Hoshino, M., and Kaibuchi, K. 2005. PAR-6-PAR-3 mediates Cdc42-induced Rac activation 
through the Rac GEFs STEF/Tiam1. Nat. Cell Biol. 7: 270277.

Noctor, S.C., Martinez-Cerdeno, V., Ivic, L., and Kriegstein, A.R. 2004. Cortical neurons arise in symmetric and asymmetric division zones and migrate through specific phases. Nat. Neurosci. 7: 136-144.

Ozcan, U., Ozcan, L., Yilmaz, E., Duvel, K., Sahin, M., Manning, B.D., and Hotamisligil, G.S. 2008. Loss of the tuberous sclerosis complex tumor suppressors triggers the unfolded protein response to regulate insulin signaling and apoptosis. Mol. Cell 29: 541-551.

Potter, C.J., Pedraza, L.G., and Xu, T. 2002. Akt regulates growth by directly phosphorylating Tsc2. Nat. Cell Biol. 4: 658-665.

Sahin, M., Greer, P.L., Lin, M.Z., Poucher, H., Eberhart, J., Schmidt, S., Wright, T.M., Shamah, S.M., O'Connell, S., Cowan, C.W., et al. 2005. Eph-dependent tyrosine phosphorylation of ephexin 1 modulates growth cone collapse. Neuron 46: 191-204.

Sarbassov, D.D., Ali, S.M., and Sabatini, D.M. 2005. Growing roles for the mTOR pathway. Curr. Opin. Cell Biol. 17: 596603.

Schwamborn, J.C. and Puschel, A.W. 2004. The sequential activity of the GTPases Rap1B and Cdc42 determines neuronal polarity. Nat. Neurosci. 7: 923-929.

Shaw, R.J., Bardeesy, N., Manning, B.D., Lopez, L., Kosmatka, M., DePinho, R.A., and Cantley, L.C. 2004a. The LKB1 tumor suppressor negatively regulates mTOR signaling. Cancer Cell 6: 91-99.

Shaw, R.J., Kosmatka, M., Bardeesy, N., Hurley, R.L., Witters, L.A., DePinho, R.A., and Cantley, L.C. 2004b. The tumor suppressor LKB1 kinase directly activates AMP-activated kinase and regulates apoptosis in response to energy stress. Proc. Natl. Acad. Sci. 101: 3329-3335.

Shelly, M., Cancedda, L., Heilshorn, S., Sumbre, G., and Poo, M.M. 2007. LKB1/STRAD promotes axon initiation during neuronal polarization. Cell 129: 565-577.

Shi, S.-H., Jan, L.Y., and Jan, Y.-N. 2003. Hippocampal neuronal polarity specified by spatially localized mPar3/mPar6 and PI 3-kinase activity. Cell 112: 63-75.

Sosa, L., Dupraz, S., Laurino, L., Bollati, F., Bisbal, M., Caceres, A., Pfenninger, K.H., and Quiroga, S. 2006. IGF-1 receptor is essential for the establishment of hippocampal neuronal polarity. Nat. Neurosci. 9: 993-995.

Stocker, H., Radimerski, T., Schindelholz, B., Wittwer, F., Belawat, P., Daram, P., Breuer, S., Thomas, G., and Hafen, E. 2003. Rheb is an essential regulator of $S 6 \mathrm{~K}$ in controlling cell growth in Drosophila. Nat. Cell Biol. 5: 559-565.

Tavazoie, S.F., Alvarez, V.A., Ridenour, D.A., Kwiatkowski, D.J., and Sabatini, B.L. 2005. Regulation of neuronal morphology and function by the tumor suppressors Tscl and Tsc2. Nat. Neurosci. 8: 1727-1734.

Tee, A.R., Fingar, D.C., Manning, B.D., Kwiatkowski, D.J., Cantley, L.C., and Blenis, J. 2002. Tuberous sclerosis complex-1 and -2 gene products function together to inhibit mammalian target of rapamycin (mTOR)-mediated downstream signaling. Proc. Nat1. Acad. Sci. 99: 1357113576.

Tee, A.R., Manning, B.D., Roux, P.P., Cantley, L.C., and Blenis, J. 2003. Tuberous sclerosis complex gene products, Tuberin and Hamartin, control mTOR signaling by acting as a GTPase-activating protein complex toward Rheb. Curr. Biol. 13: $1259-1268$.

Wullschleger, S., Loewith, R., and Hall, M.N. 2006. TOR signaling in growth and metabolism. Cell 124: 471-484.

Xiao, J. and Monteiro, M.J. 1994. Identification and character- ization of a novel (115 kDa) neurofilament-associated kinase. J. Neurosci. 14: 1820-1833.

Yoshimura, T., Kawano, Y., Arimura, N., Kawabata, S., Kikuchi, A., and Kaibuchi, K. 2005. GSK-3 $\beta$ regulates phosphorylation of CRMP-2 and neuronal polarity. Cell 120: 137-149.

Zhang, Y., Gao, X., Saucedo, L.J., Ru, B., Edgar, B.A., and Pan, D. 2003. Rheb is a direct target of the tuberous sclerosis tumour suppressor proteins. Nat. Cell Biol. 5: 578-581.

Zhu, Y., Romero, M.I., Ghosh, P., Ye, Z., Charnay, P., Rushing, E.J., Marth, J.D., and Parada, L.F. 2001. Ablation of NF1 function in neurons induces abnormal development of cerebral cortex and reactive gliosis in the brain. Genes \& Dev. 15: 859-876. 


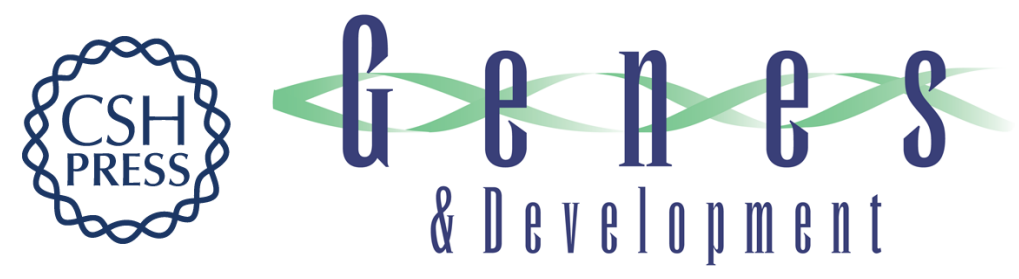

\section{Tuberous sclerosis complex proteins control axon formation}

Yong-Jin Choi, Alessia Di Nardo, loannis Kramvis, et al.

Genes Dev. 2008, 22:

Access the most recent version at doi:10.1101/gad.1685008

\section{Supplemental http://genesdev.cshlp.org/content/suppl/2008/09/16/22.18.2485.DC1 \\ Material}

Related Content

References

Email Alerting Service

\section{License}

The Tsc1Tsc2 complex influences neuronal polarity by modulating TORC1 activity and SAD levels

Jill Wildonger, Lily Yeh Jan and Yuh Nung Jan

Genes Dev. September , 2008 22: 2447-2453

This article cites 46 articles, 12 of which can be accessed free at:

http://genesdev.cshlp.org/content/22/18/2485.full.html\#ref-list-1

Articles cited in:

http://genesdev.cshlp.org/content/22/18/2485.full.html\#related-urls

Receive free email alerts when new articles cite this article - sign up in the box at the top right corner of the article or click here.

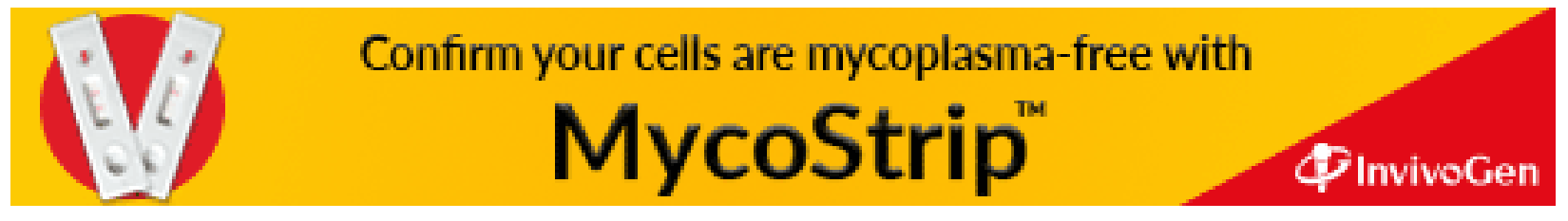

